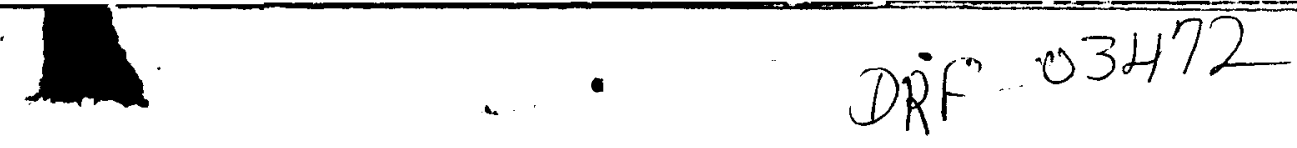

\title{
EVOLUTION OF THE EARTH'S ATMOSPHERE
}

"We living things are a late outgrowth of the metabolism of our Galaxy. The carbon that enters so importantly into our somposition was cooked in the remote past in a dying star. From it at lower temperatures nitrogen and oxygen were formed. These, our indispensable elements, were spewed out into space in the exhalations of red giants and such stellar catastrophes as supernovae, there to be mixed with hydrogen, to form eventually the substance of the sun and planets, and ourselves. The waters of ancient seas

set the pattern of ions in our blood. The ancient
-George wald

set the pattern of ions in our blood. The ancient
-George Wald nitrogen, $21 \%$ oxygen and $1 \%$ argon, with traces of carbon dioxide, water vapor and ozone. The atmospheres of Mars and Venus, on the other hand, are predominantly composed of carbon dioxide, while those of Jupiter and Saturn contain mainly hydrogen, helium, methane and ammonia. Such a wide variety in the composition of the atmospheres of the planets afvears most intrijuing when one considers that all nine planets were probably formed at the same time and out of the same chemicaily homogeneous mixture of gas and dust; that is, the primitive solar nebula:

The most likely explanation for this diversity in composition seems to be that the planetary atmospheres have underqone

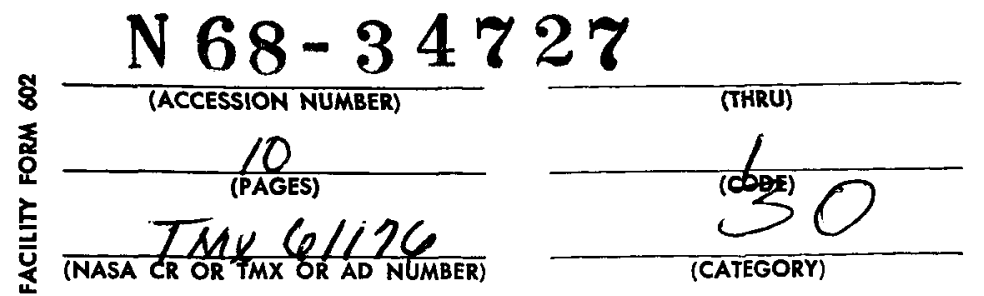
GPO PRICE $\$$ CSFTI PRICE(S) $\$$ 
important evolutionary changes during their long history of about 4.5 billion years. On the earth, the evidence for such changes is manifold. Ever since Louis Pasteur demonstrated that spontaneous generation of life does not take place at the present time, it has become obvious that if life originated on the earth, as it probably did, the atmospheric conditions in the early history of the planet musc have been quite different in order for the chemical evolution to have taken place. Also, it appears that relitive to the sun, the earth is deficient by several orders of magnitude, not only in hydrogen and helium, which being the lighest can be assumed to have escaped from the gravitational field of the earth, but also in carbon, nitrogen, and the cosmically abundant rare gases; namely, Ne, $\mathrm{A}^{36}, \mathrm{Kr}$ and $\mathrm{Xe}$, (Table 1). A comparison of the deficiency factors of these elements with those of non-volatiles like $\mathrm{Na}, \mathrm{Mg}, \mathrm{Al}$ and $\mathrm{Si}$, strongly suggests that the earth lost all elements which could have been gaseous at temperatures of a few hundred degrees during the very early history of the planet. The relatively small amounts of carbon, hydrogen, nitrogen and oxygen now present in the crust, hydrosphere and atmosphere were probably outgassed from the interior and accumulated slowly during the lifetime of the earth. 
Both biochemical arguments and geoloaical evidence, therefore, strongly suggest that the atmosphere of the earth has undergone major evolutionary changes during its long history. Whether the atmosi heres of llars and Venus have also gone through similar stages of evolution is a question which cannot be answered without more knowledge about their composition. Future in situ exploration of these planets and a search for the rare gases, especially $\mathrm{Ne}$, which is deficient on earth by a factor of $10^{10}$, will provide important clues to the origin of these atmospheres. For the earth, there is convincing evidence that the present atmosphere and hydrosphere arose largely from the interior via volcanic emanations. But the sequence of events which led to present-day composition of $\mathrm{N}_{2}$ and $\mathrm{O}_{2}$ has yet to be established. What is the history of the volatiles now present at the surface of the earth? Has the $C, N, O$ and $H$ always been in the form of $\mathrm{CO}_{2}, \mathrm{~N}_{2}$, and $\mathrm{H}_{2} \mathrm{O}$ and $\mathrm{H}_{2}$, or did carbon and nitrogen combine with hydrogen early in the earth's history to form $\mathrm{CH}_{4}$ and $\mathrm{NH}_{3}$ ? Under what atmospheric conditions did life originate on earth and how did the appearance of life change the atmosphere? These are some of the basic questions which must be answered in order to paint a coherent pict'ire of the evolution of the earth's atmosphere. 
Opinions on these questions are many and varied; sometimes they are almost diametrically opposed. The Oparin-Urey theory of the origin of life on the earth, supported by the laboratory experiments of Miller and more recently of Ponnamperuma, suggest a primitive atmosphere composed mainly of $\mathrm{CH}_{4}$ with small amounts of $\mathrm{NH}_{3}, \mathrm{H}_{2}$ and $\mathrm{H}_{2} \mathrm{O}$ vapor. On the other hand, the school of thought led by Aluelson, and supported also by laboratory experiments on the synthesis of amino acids, holds that the early atmosphere of the earth was made up of $\mathrm{CO}_{2}, \mathrm{CO}, \mathrm{H}_{2}, \mathrm{~N}_{2}$ and $\mathrm{H}_{2} \mathrm{O}$ vapor.

Geologists are also divided on the subject. Holland has presented a model for the evolution of the atmosphere in which, during the very early stage aftor the formation of the earth and at the comincement of outgassing, the dominant gases could have been $\mathrm{CH}_{4}$ and $\mathrm{H}_{2}$ ' provided free hydrogen did not 'escape' as rapidly as it does today. Rubey, on the other hand, believes that the early atmosphere was probably made of $\mathrm{CO}_{2}$ and $\mathrm{N}_{2}$ because not enough hydrogen was available to keep $\mathrm{CH}_{4}$ from converting into $\mathrm{CO}_{2}$. Holland's model is supported by the calculations of Rasool and McGovern, who have investigated the thermal properties of model primitive atmospheres of the earth. They a find that in/99\% $\mathrm{CH}_{4}-1 \% \mathrm{H}_{2}$ atmosphere the average exospheric 
temperature may be as low as $650^{\circ} \mathrm{K}$ (cf. present day value $1500^{\circ} \mathrm{K}$ ). making the escape of hydrogen a relatively sluw phenomenon. However, Abelson has argued that if methane were abundant in the primitive atmosphere, the earliest rocks should contain unusual amounts of organic matter, which apparently is not the case.

Despite the disagreement over the composition of the primitive atmosphere, it is almost certain that it was devoid of free oxygen. How and when did free oxygen then become a major constituent of the atmosphere? The late Lloyd V. Berkner and L.C. Marshall have presented detailed calculations of the photochemistry of $\mathrm{H}_{2} \mathrm{O}$, indicating that free oxygen was limited to $\sim 0.1 \%$ of the present atmospheric level during the entire prebiological history, accumulating slowly to the present amount since the start of photosynthesis about 800 million years ago. This is probably the most widely accepted explanation for the growth of oxygen in the earth's atmosphere. However, difficulties arise when one attempts to construct an evolutionary model of the atmosphere which would be consistent from the prebiological period to the present. For example, it is not well understood whether the small amount of $\mathrm{O}_{2}$ suggested to be present in the primitive atmosphere would not be sufficient to rapldly oxidize $\mathrm{CH}_{4}$ and 
$\mathrm{NH}_{3}$ into $\mathrm{CO}_{2}$ and $\mathrm{N}_{2}$. It may be that the production of even this small amount of free oxygen in the upper atmosphere by the photodi-sociation of water vaopr was inhibited by the presence of $\mathrm{NH}_{3}$ itself.

Some of these important questions will be discussed in the forthcoming interdisciplinary symposium on the Evolution of the Earth's Atmosphere to be held during the Annual Meetings of the AAAS in New York City, under the chairmanship of Harrison Brown of the California Institute of Technology.

Because of the diversity of disciplines involved, an attempt has been made to bring together geologists, biologists, meteorologists and astrophysicists, with the hope of obtaining fresh ideas and a broader perspective on the subject. The program includes the following presentations: "Nature of the Early Atmosphere and Ocean" (P. Cloud), "Gravitational Escape of Hydrogen from Model Primitive Atmospheres" (S.I. Rasool and W.E. McGovern), "Simulated Atmospheres and Models of the Synthesis of the First Organisms" (S.W. Fox), "Evolution of $\mathrm{N}_{2}-\mathrm{O}_{2}$ Atmosphere on Earth" (L.C. Marshall), Atmospheric $\mathrm{CO}_{2}$, Past and Present" (H.D. Holland), followed by "Summary and Critique" (H. Brown). There is a chance that this discussion may bring 
us a step closer to understanding the nature of the ancient atmospheres that molded our metabolism.

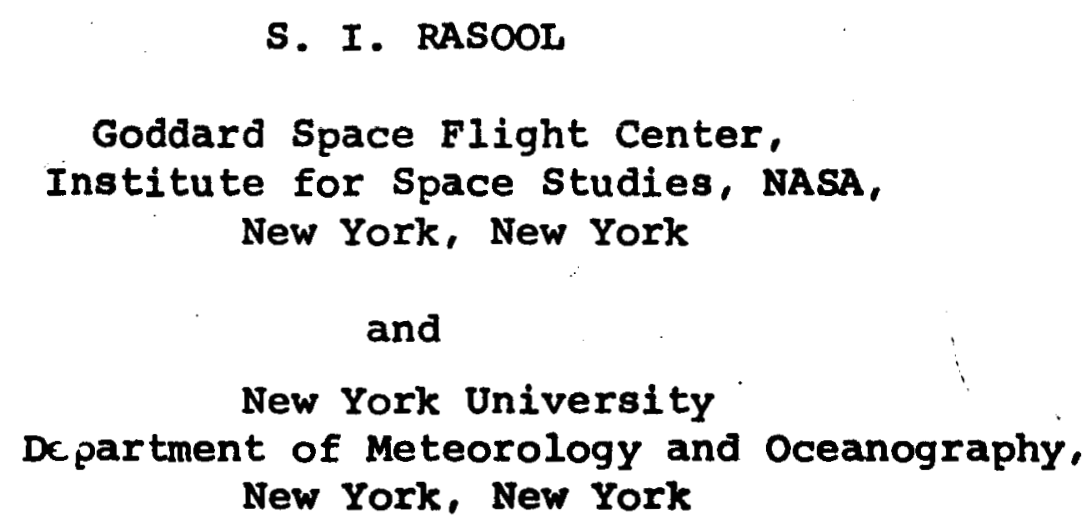

S. I. RASOOL

Goddard Space Flight Center, Institute for Space Studies, NASA, New York, New York

and

New York University

Department of Meteorology and Oceanography, New York, New York 
TABLE 1.

(ATOMS/10,000 ATOMS Si)

WHOLE EARTH*

(a)

H

He

c

N

O

Ne

$\mathrm{Na}$

Mg

Al

$\mathbf{S i}$

$A^{36}$

$\mathrm{Kr}$

Xe
$6 \times 10^{-8}$

$5.9 \times 10^{-4}$

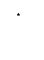

\section{SOLAR SYSTEM**}

(b)

$2.6 \times 10^{8}$

$2.1 \times 10^{7}$

135,000

24,400

236,000

23,000

632

$10,50^{\circ}$

851

10,000

2,280

0.69

0.07
DEF ICIENCY FACTOR

$\log (b / a)$

6.0

13.8

4. 0

5.1

0.8

10.3

$\sim 0$

$\sim 0$

$\sim 0$

0

6.6

$5 \times 10^{-9}$ 